\title{
Warum heute Frauenkanzlei
}

\author{
Maria Demirci \\ Rechtsanwältin, München
}

Die Entscheidung, in einer reinen Frauenkanzlei zu arbeiten, habe ich aus folgenden Gründen bewusst getroffen:

Bereits während des Studiums und zu Beginn meines Referendariats stand für mich fest, dass ich meinen Schwerpunkt im Familienrecht und im Erbrecht setzen möchte.

Während meines Referendariats wurde von der Universität München ein Vortragsabend mit dem Titel „Frauen, Juristinnen und ihre Zukunft“ angeboten. Zu diesem Vortrag waren mehrere Frauen eingeladen, um über die Thematik zu referieren, darunter auch die Vizepräsidentin des Deutschen Juristinnenbundes und meine heutige Kollegin, Frau Renate Maltry.

An diesem Tag wurde ich zum ersten Mal auf Frau Maltry und ihre Kanzlei aufmerksam gemacht.

Mich faszinierte vor allem, dass Frau Maltry sich in ihrer Kanzlei nur auf die zwei Bereiche spezialisiert hat. Des Weiteren war es für mich beeindruckend zu hören, dass in dieser Kanzlei Familie und Karriere, im sonst sehr stressigen Anwaltsberuf, möglich erscheinen soll und auch möglich gemacht wird.

Nach diesem Vortragsabend entschied ich mich daher, meine Referendarsstation in der Kanzlei Maltry Rechtsanwältinnen zu absolvieren.

Während dieser Zeit wurde mir bewusst, dass in einer reinen Frauenkanzlei anders gearbeitet wird.

Bereits die Arbeitsatmosphäre ist eine andere. Es findet ein reger fachlicher Austausch zwischen den Anwältinnen statt. Es wird nicht nur auf die fachliche Kompetenz Wert gelegt. Auch auf unternehmerisches Know-how, Soft Skills und Kanzleimanagement wird geachtet und es wird gefördert. Es geht darum, die Rechte und Interessen von Frauen sachgerecht und kompetent durchzusetzen. Es zeichnet uns auch aus, dass wir nicht nur immer Recht haben wollen und alles durchsetzen möchten. Bei jedem Fall ist es uns wichtig, den Menschen bzw. die Frau, die hinter diesem Fall steht, nicht zu vergessen. Frauen haben nun einmal eine andere Art der Kommunikation und ein gewisses Einfühlungsvermögen. Oft kommen Mandantinnen zu uns, welche von männlichen Kollegen vertreten wurden, und berichten, dass sie nicht zufrieden sind. Meist geht es gar nicht um die fachliche Kompetenz, sondern um das fehlende Einfühlungsvermögen.

Auch rein optisch unterscheidet sich die Kanzlei Maltry Rechtsanwältinnen von anderen Kanzleien.

Die Räume sind hell, offen und freundlich eingerichtet, im Gegensatz zur klassischen Anwaltskanzlei, welche meist mit schweren dunklen Schreibtischen ausgestattet ist und in der eine eher düstere Atmosphäre ausgestrahlt wird.

Für den besonders sensiblen Bereich des Familienrechts ist eine solche Atmosphäre jedoch kontraproduktiv. Die Man- danten kostet der Weg zur Familienrechtsanwältin Überwindung. Sie müssen ihrer Anwältin bzw. ihrem Anwalt einen großen Teil ihres Privatlebens preisgeben. Dafür ist eine entspannte, angenehme und freundliche Atmosphäre förderlich. Dies alles habe ich in dieser Kanzlei gefunden.

Nach neunmonatiger Referendarsstation stand für mich dann fest:

Nach dem Abschluss des 2. Staatsexamens möchte ich meine berufliche Karriere in dieser Kanzlei beginnen.

Im Mai habe ich mein „einjähriges Kanzleijubiläum“ gefeiert. Ich blicke auf dieses Jahr zurück und sage mir: Es gibt noch viel zu tun!

Meine Kollegin und Kanzleigründerin, Frau Renate Maltry, lehrte mich, dass es mit der rein fachlichen Fort- und Weiterbildung nicht getan ist. Wir Frauen können neben und durch unseren Beruf dazu beitragen, frauenpolitisch zu arbeiten.

Natürlich denken viele, dass es heutzutage für Frauen eigentlich kein Problem sein sollte, mit den Männern im Beruf und im Allgemeinen Schritt zu halten.

Viele Kolleginnen sagen sich, dass in unserem Berufsfeld bereits genug für Frauen getan wurde.

Dem ist jedoch nicht so. Wir Frauen sind immer noch in vielen Bereichen unterrepräsentiert und werden benachteiligt. Bei der diesjährigen Tagung der EWLA in Brüssel wurde mir bewusst, dass vieles noch im Argen ist. Wir Frauen machen keine Fort-, sondern erschreckenderweise Rückschritte. Es ist an uns, die Initiative zu ergreifen, und das fortzuführen, was die Frauenbewegung in Gang gesetzt hat. Es geht nicht darum, Frauen um jeden Preis an die Macht zu stellen. Es geht auch nicht um Geschlechterkampf. Es geht einzig und allein um Gleichberechtigung.

Heutzutage ist zwar der Anteil weiblicher Jurastudentinnen und Rechtsanwältinnen erheblich gestiegen. Bereits als ich das Studium im Jahre 2002 aufnahm, waren mehr als 50 Prozent der Studenten weiblich. Wo sind diese Frauen jedoch alle geblieben? Wir verschwinden oft in den „Randbereichen “ oder schrecken vor einer Karriere zurück, da ansonsten eine Familienplanung nicht möglich ist.

Die Kanzleigründerin beweist seit 26 Jahren das Gegenteil und gibt den Kolleginnen die Möglichkeit, Kind und Karriere unter einen Hut zu bekommen. Für mich ist sie die Vorreiterin und verkörpert mein Ideal: die Vereinbarkeit von Beruf und Familie.

Natürlich ist diese Doppelbelastung nicht einfach und mit vielen Entbehrungen verbunden. Wir dürfen uns aber nicht davor scheuen, diese Doppelbelastung einzugehen. Wir müssen dabei auch lernen, Verantwortung abzugeben, indem wir Männer in die Pflicht nehmen. Dadurch würden wir die Doppelbelastung reduzieren.

Wir sollten auch lernen, Risiken einzugehen und mehr unternehmerisch denken. Auch das vermisse ich bei manchen 
jungen Kolleginnen. Bloßes Sicherheitsdenken wird uns karrieretechnisch nicht weiterbringen.

Dieser Herausforderung muss „Frau“ sich stellen, ansonsten werden sich die Rollenstereotype auch in 100 Jahren nicht verändern.
Nicht nur die Männer stehen uns im Weg, sondern oftmals wir selbst! Diese Erkenntnis ist erschreckend. Nichts im Leben ist einfach und nichts wird einem geschenkt.

Wer aber nicht wagt, der nicht gewinnt.

\section{Meine ersten 100 Tage als Volljuristin: Arbeiten in der Hamburg Port Authority}

\section{Ass. jur. Kirsten Junga-Suhr \\ Wissenschaftliche Mitarbeiterin, Hamburg}

November 2008 - endlich, ich hatte es geschafft: Nach einem dreiviertel Jahr bewerben, Absagen verarbeiten, sich immer wieder neu motivieren, hatte ich die Zusage für die Stelle als wissenschaftliche Angestellte bei der Hamburg Port Authority.

Seit Januar 2009 arbeite ich also in der Rechtsabteilung (öffentlich-rechtliches Referat) der Hamburg Port Authority. Sie wurde 2005 im Zuge der Zusammenführung der hafenbezogenen Zuständigkeiten verschiedener Hamburger Behörden als Anstalt öffentlichen Rechts gegründet und bietet Hafenmanagement aus einer Hand.

Mein Tätigkeitsbereich erstreckt sich über das Hafenverkehrs- und Schifffahrtsrecht über das Umweltrecht bis hin zum Fachplanungsrecht. Ich denke, dass ich nichts Falsches behaupte, wenn ich sage, dass dies nicht gerade Themen sind, mit denen man sich bereits während der Ausbildung ausgiebig beschäftigt hat. Aber es hat sich der Spruch bewahrheitet, dass wir während unserer Ausbildung das „Handwerkszeug“ lernen, mit dem es gelingt, sich schnell in jedes Rechtsgebiet einzuarbeiten. Ich kann das wirklich nur bestätigen.

Auch konnte ich auf das verwaltungsprozessuale Ausbildungswissen zurückgreifen, wenn es um die Prozessbegleitung geht. Das ist sehr hilfreich. Denn dann hat man neben den vielen neuen Themengebieten auch das Gefühl, auf bereits verankertes Wissen zurückgreifen zu können.

Auch wenn ich mittlerweile routinierter bin, so werde ich doch immer wieder - viele von euch werden das kennen - mit Fragestellungen konfrontiert, deren Lösung man in keinem Lehrbuch findet. In solchen Situationen kommt es dann wirklich darauf an, dass man die Materie verstanden hat und in der Lage ist, mit Hilfe von ähnlich gelagerten und bereits entschiedenen Fällen die richtige Lösung zu entwickeln. Gerade in der Anfangszeit ist es sehr hilfreich, dass ich mich jederzeit an meine Kolleginnen und Kollegen wenden kann. In der Rechtsabteilung der HPA arbeiten sechs Juristinnen und zwei Juristen; in meinem Referat sind wir zu viert.

Da ich verschiedene Fachabteilungen im Hause berate, habe ich mit den unterschiedlichsten Kund(inn)en zu tun und damit auch mit unterschiedlichen juristischen Fragestellungen. Das macht die Tätigkeit sehr abwechslungsreich. Die Zusammenarbeit mit den Kund(inn)en finde ich sehr interessant, obgleich ich darauf während meiner Ausbildung eigentlich nicht vorbereitet wurde. Während der Ausbildung gab es überwiegend feststehende Sachverhalte, die es zu analysieren galt. Doch jetzt muss ich zum Beispiel in Funktion als Widerspruchsbehörde auch den Sachverhalt ermitteln oder Nichtjurist(inn)en rechtliche Aspekte verständlich erklären. Hier kommt es in erheblichem Maße auf die sogenannten Soft Skills und weniger auf die Examensnote an.

Zudem bekomme ich aufgrund der Verbindung zur Hamburger Verwaltung interessante Einblicke in Gesetzgebungsverfahren sowie das Zusammenspiel von Politik und Verwaltung.

Überrascht hat mich ehrlich gesagt die enorme Fülle europarechtlicher Vorgaben besonders auf dem Gebiet des Hafenverkehrs- und Schifffahrtsrechts. Europa bekommt für mich dadurch eine ganz neue Bedeutung bzw. mir wird die Bedeutung erst jetzt richtig bewusst. Aus juristischer Sicht unglaublich spannend finde ich die Mitarbeit bei der Umsetzung europäischer Richtlinien.

Als ich schließlich das erste Mal meine Arbeitgeberin vor dem Verwaltungsgericht vertrat und das Gericht zu Beginn sagte „Und für die Beklagte erscheint Frau Junga-Suhr“, wurde mir erstmals richtig bewusst, dass ich nach jahrelanger Ausbildung samt zwei Examina nun endlich richtig im Arbeitsleben als Volljuristin angekommen bin. Das ist ein sehr gutes Gefühl! 